Wasserstoffentwicklung ein dicker weißer Niederschlag der Zusammensetzung $\mathrm{Al}(\mathrm{NHR})_{3}$ aus:

$$
\mathrm{AlH}_{3}+3 \mathrm{NH}_{2} \mathrm{R} \rightarrow \mathrm{Al}(\mathrm{NHR})_{3}+3 \mathrm{H}_{2} .
$$

Die Substanz stellt ein Methylderivat des Aluminiumtriamids $\mathrm{Al}\left(\mathrm{NH}_{2}\right)_{3}{ }^{6}$ dar und dürfte wie dieses hochpolymer sein [Ersatz der beiden an $\mathrm{Al}$ gebundenen $\mathrm{H}$-Atome in (7) durch NHR-Gruppen], da sie in Äther und Benzol unlöslich und nicht-flüchtig ist. Sie konnte bei der geschilderten Arbeitsweise nicht völlig rein erhalten werden (gefunden im besten Falle $\mathrm{Al}: \mathrm{N}=1: 2.86$ statt theoretisch $1: 3$ ), da unter den gewählten Versuchsbedingungen teilweise auch schon das zweite Wasserstoffatom des Methylamins mit dem Aluminiumwasserstoff in Reaktion tritt [vgl. (5)]. Beim Erhitzen auf $200^{\circ}$ im Vakuum spaltet die Verbindung $\mathrm{Al}(\mathrm{NHR})_{3}$ gemäß dem Schema

$$
2 \mathrm{Al}(\mathrm{NHR})_{3} \rightarrow \mathrm{Al}_{2}(\mathrm{NR})_{3}+3 \mathrm{NH}_{2} \mathrm{R}
$$

Methylamin ab, wobei das theoretische Verhältnis $\mathrm{Al}: \mathrm{N}$ $=1: 1,5 \mathrm{im}$ Kondensationsprodukt nicht ganz erreicht wird (gef. z. B. $1: 1,68$ und $1: 1,78$ ).

Nimmt man die Umsetzung von Aluminiumwasserstoff mit überschüssigem Methylamin nicht bei Zimmertemperatur, sondern bei tiefer Temperatur, z. B. $-50^{\circ}$ vor und destilliert den Äther und das überschüssige Methylamin bei der gleichen niedrigen Temperatur ab, so hinterbleibt das schon erwähnte feste, weiße Di-aminat $\mathrm{AlH}_{3} \cdot 2 \mathrm{NH}_{9} \mathrm{R}$ (9), das oberhalb von $-40^{\circ}$ in $\mathrm{AlH}(\mathrm{NHR})$, übergeht (10). Die Bildung von $\mathrm{Al}(\mathrm{NHR})_{3}$ aus Aluminiumwasserstoff und überschüssigem Methylamin verläuft hiernach mit großer Wahrscheinlichkeit in Analogie zur Bildung von $\mathrm{Al}\left(\mathrm{NH}_{2}\right)_{3}$ aus Aluminiumwasserstoff und Ammoniak ${ }^{6}$ gemäß dem folgenden Schema über die Zwischenstufen $\mathrm{AlH}_{2}(\mathrm{NHR})$ und $\mathrm{AlH}(\mathrm{NHR})_{2}$ :

$$
\begin{gathered}
\mathrm{AlH}_{3} \stackrel{+2 \mathrm{NH}_{2} \mathrm{R}}{\longrightarrow} \mathrm{AlH}_{3} \cdot 2 \mathrm{NH}_{2} \mathrm{R} \stackrel{-\mathrm{H}_{2}}{\longrightarrow} \mathrm{AlH}_{2}(\mathrm{NHR}) \cdot \mathrm{NH}_{2} \mathrm{R} \\
\stackrel{-\mathrm{H}_{2}}{\longrightarrow} \mathrm{AlH}(\mathrm{NHR})_{2} \stackrel{+\mathrm{NH}_{2} \mathrm{R}}{\longrightarrow} \mathrm{AlH}(\mathrm{NHR})_{2} \cdot \mathrm{NH}_{2} \mathrm{R} \\
\stackrel{-\mathrm{H}_{2}}{\longrightarrow} \mathrm{Al}(\mathrm{NHR})_{3} .
\end{gathered}
$$

Analoge Di-aminate wie mit Methylamin (9) werden bei der Umsetzung von Aluminiumwasserstoff mit überschüssigem Trimethylamin 7 , Dimethylamin ${ }^{8}$ und Ammoniak ${ }^{6}$ gebildet.

6 E. Wiberg u. A. M a y, Z. Naturforschg. 10 b, 230 [1955].

7 E. Wiberg, H. Graf u. R. Usón, Z. anorg. allg. Chem. 272, 221 [1953].

8 E. Wiberg u. A. May, Z. Naturforschg. 10 b, 234 [1955].

\section{Über die Umsetzung von Aluminiumwasserstoff mit Ammoniak und Aminen}

\section{Zur Kenntnis einer Verbindungsreihe $\mathrm{AlH}_{3-\mathrm{n}}\left[\mathbf{N}\left(\mathrm{CH}_{3}\right)_{2}\right]_{\mathrm{n}}$}

Von Egon Wiberg und Adolf May

Institut für Anorganische Chemie der Universität München

(Z. Naturforschg. 10 b, 234-235 [1955]; eingeg. am 7. März 1955)

In Analogie zur Bildung eines isobutylen-isosteren Dimethylborazens $\mathrm{BH}_{2}-\mathrm{NR}_{2}$ aus Borwasserstoff und Dimethylamin läßt sich aus äquimolekularen Mengen von Aluminiumwasserstoff und Dimethylamin bei Zimmertemperatur ein Dimethyl-alazen $\mathrm{AlH}_{2}=\mathrm{NR}$ ? gewinnen, das das leichtere Borhomologe an Dimerisationsfreudigkeit übertrifft. Durch Einführung von $\mathrm{NR}_{2}$-Gruppen in das Molekül wird wie im Falle des Borazens die Dimerisationsneigung geschwächt.

Bei $-40^{\circ}$ bildet sich aus Aluminiumwasserstoff und überschüssigem Dimethylamin ein Di-aminat $\mathrm{AlH}_{3} \cdot 2 \mathrm{NHR}_{2}$, das oberhalb von $-20^{\circ}$ Wasserstoff abspaltet.

Bei der Umsetzung von Borwasserstoff mit Dimethylamin im Molverhältnis $\mathrm{BH}_{3}: \mathrm{NHR}_{2}=1: 1$ entsteht ein mit dem Dimethyl-äthylen (Isobutylen) $\mathrm{CH}_{2}=\mathrm{CR}_{2}$ isosteres Dimethyl-borazen $\mathrm{BH}_{2}-\mathrm{NR}_{2}$ :

$$
\mathrm{BH}_{3}+\mathrm{NHR}_{2} \rightarrow \mathrm{BH}_{3} \cdots \mathrm{NHR}_{2} \rightarrow \mathrm{BH}_{2}-\mathrm{NR}_{2}+\mathrm{H}_{2},
$$

das wie jenes ungesättigten Charakter besitzt und dementsprechend zur Dimerisation neigt ${ }^{1}$. Einführung von $\mathrm{NR}_{2}$-Gruppen in das Molekül führt zur Schwächung der Polymerisationstendenz, wie aus dem rein monomeren Charakter der Dimethylamino-Derivate $\mathrm{BH}\left(\mathrm{NR}_{2}\right)-\mathrm{NR}_{2} 2$ und $\mathrm{B}\left(\mathrm{NR}_{2}\right)_{2}-\mathrm{NR}_{2}{ }^{3}$ hervorgeht. Es war nun von Interesse, nachzuprüfen, ob auch homologe „Alazene“ darstellbar sind und ob diese eine entsprechende Abstufung ihrer Polymerisationsfreudigkeit erkennen lassen. Das Experiment bejahte beide Fragen.

\section{Die Verbindung $\mathrm{AlH}_{2}\left(\mathrm{NR}_{2}\right)$}

Gibt man bei $-40^{\circ}$ ätherische Lösungen von Aluminiumwasserstoff und Dimethylamin im Molverhältnis $\mathrm{AlH}_{3}: \mathrm{NHR}_{2}=1: 1$ zusammen, so ist äußerlich keine Reaktion zu beobachten. Beim langsamen Erwärmen auf Zimmertemperatur setzt zwischen $-20^{\circ}$ und $-10^{\circ}$ eine geringe Gasentwicklung ein, die bei $0^{\circ}$ recht lebhaft wird und bei Zimmertemperatur rasch zu der nach der Gleichung

$$
\mathrm{AlH}_{3}+\mathrm{NHR}_{2} \rightarrow \mathrm{AlH}_{2}\left(\mathrm{NR}_{2}\right)+\mathrm{H}_{2}
$$

zu erwartenden Wasserstoffmenge führt.

Destilliert man nach Beendigung der Reaktion den Äther im Vakuum bei Zimmertemperatur ab und erwärmt den verbleibenden weißen Rückstand im Hochvakuum, so sublimiert das Reaktionsprodukt zwischen 40 und $60^{\circ} \mathrm{ab}$, um sich in Form glänzender, farbloser, stark lichtbrechender Prismen an den kälteren Teilen der Sublimationsapparatur abzuscheiden, von wo es mit dem Fön leicht in eine mit flüssiger Luft gekühlte Vorlage übergetrieben werden kann. Seine Zusammensetzung entspricht nach der Aluminium-, Wasserstoff- und Dimethylamin-Bestimmung erwartungsgemäß der Bruttoformel $\mathrm{AlH}_{2} \mathrm{NR}_{2}$, wonach das gesuchte Dimethyl-alazen vorliegt. Gemäß der ebullioskopischen Molekulargewichtsbestimmung in Äther ist die Verbindung dimer (M gef.: 158: M ber.: 146), entsprechend einer Formel

1 E. Wiberg, A. Bolz u. P. B u chheit, Z. anorg. Chem. 256, 285 [1948]; A. B. B urg u. C. L. R a n d o lp h jr., J. Amer. chem. Soc. 73, 953 [1951].

2 E. Wi berg u. A. B olz, Z. anorg. Chem. 257, 131 [1948]; A. B. Burg u. C. L. Randolph jr., J. Amer. chem. Soc. 73, 953 [1951].

3 E. Wi berg u. K. S chuster, Z. anorg. Chem. 213, 77 [1933]; A. B. B urg u. C. L. R a n dolph jr., J. Amer. chem. Soc. 73, 953 [1951]. 
<smiles>[H][Y11]1([H])[R17][Y11]1([H])[H]</smiles>

Die Kristalle schmelzen unzersetzt bei $89-90^{\circ}$, rauchen an der Luft (gelegentliche starke Erwärmung und Zersetzung unter Braunfärbung), reagieren mit Wasser heftig unter Wasserstoffentwicklung, lösen sich leicht in Benzol und Äther und zerfallen beim Erhitzen über den Schmelzpunkt hinaus zuerst unter Braunfärbung der Schmelze, dann $\left(\sim 130^{\circ}\right)$ unter rascher Ausscheidung dunkler Flocken.

Wie aus dem dimeren Charakter der Verbindung hervorgeht, besitzt das Dimethyl-alazen $\mathrm{AlH}_{2} \mathrm{NR}_{2}$ wie das homologe Borazen $\mathrm{BH}_{2} \mathrm{NR}_{2}$ die Neigung, sich zu polymerisieren. Und zwar ist seine Polymerisationstendenz noch ausgeprägter als bei diesem, da sich seine dimere Form nicht wie im Falle der analogen Borverbindung 1 mit der monomeren Form im Gleichgewicht befindet.

Erwähnt sei bei dieser Gelegenheit noch, daß das zum $N$-Dimethyl-alazen $\mathrm{AlH}_{2} \mathrm{NR}_{2}$ isomere, schon früher von E. Wiberg und A. B olz $\mathrm{z}^{4}$ beschriebene Al-Dimethylalazen $\mathrm{AlR}_{2} \mathrm{NH}_{2}$ bei höherer Temperatur $\left(134^{\circ}\right)$ schmilzt als jenes und ebenfalls dimer ist.

\section{Die Verbindung AlH( $\left(\mathrm{NR}_{2}\right)_{2}$}

Vermischt man ätherische Lösungen von Aluminiumwasserstoff und Dimethylamin bei Zimmertemperatur im Molverhältnis $\mathrm{AlH}_{3}: \mathrm{NHR}_{2}=1: 2$, so erhält man unter Wasserstoffentwicklung eine Lösung, aus der sich nach dem Abdestillieren des Lösungsmittels durch Sublimation im Hochvakuum $\left(35-50^{\circ}\right)$ eine in glänzenden, farblosen Kristallen auftretende Substanz der analytischen Zusammensetzung $\mathrm{AlH}\left(\mathrm{NR}_{2}\right)_{2}$ gewinnen läßt:

$$
\mathrm{AlH}_{3}+2 \mathrm{NHR}_{2} \rightarrow \mathrm{AlH}\left(\mathrm{NR}_{2}\right)_{2}+2 \mathrm{H}_{2} .
$$

Die Substanz, die bei $63-65^{\circ}$ schmilzt, löst sich leicht in Benzol und Äther, reagiert lebhaft mit Wasser oder Säuren und ist in der ätherischen Lösung gemäß der ebullioskopischen Mol.-Gew.-Bestimmung teils dimer, teils monomer (M gef.: 199 und 196; M ber.: für $\mathrm{AlH}\left(\mathrm{NR}_{2}\right)_{2}$ : 116, für $\left.\left[\mathrm{AlH}\left(\mathrm{NR}_{2}\right)_{2}\right]_{2}: 232\right)$, entsprechend einem Gleichgewicht:

$$
\begin{aligned}
& { }_{\mathrm{R}_{2} \mathrm{~N}}^{\mathrm{H}}>\mathrm{Al}\left\langle\underset{\substack{\mathrm{R}_{2} \\
\mathrm{R}_{2}}}{\mathrm{~N} \backslash \mathrm{Al}}\right\rangle_{\mathrm{H}}^{\mathrm{NR}_{2}}+2 \mathrm{OR}_{2} \rightleftarrows
\end{aligned}
$$

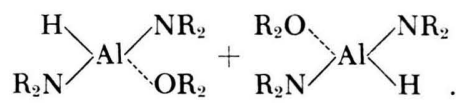

Die Einführung von $\mathrm{NR}_{2}$-Gruppen in das Alazen (2) bedingt demnach eine Herabsetzung der Polymerisations-

4 E. Wiberg u. A. B olz, FIAT-Review (deutsche Ausgabe), Bd. 24, S. 160.

5 E. Wiberg, H. Graf u. R. Usón, Z. anorg. u. allg. Chem. 272, 221 [1953]. neigung, so daß in der ätherischen Lösung der Äther die relativ schwache koordinative Brückenbindung zu lösen und sich an ihre Stelle zu setzen vermag. Jedoch ist auch hier wie im vorigen Falle die Polymerisationstendenz größer als bei der homologen Bor-Verbindung, da das analoge Borazen $\mathrm{BH}\left(\mathrm{NR}_{2}\right)-\mathrm{NR}_{2}$ ausschließlich monomer auftritt 2 .

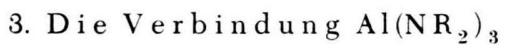

Versetzt man Aluminiumwasserstoff in ätherischer Lösung bei Zimmertemperatur mit überschüssigem Dimethylamin (Molverhältnis $\mathrm{AlH}_{3}: \mathrm{NHR}_{2}=1: 3,5$ oder 1:4) und dampft nach beendeter Wasserstoffentwicklung den Äther und Dimethylamin-Überschuß im Vakuum ab, so hinterbleibt ein weißer Rückstand, der im Hochvakuum bei $60-100^{\circ}$ absublimiert und sich in der Vorlage in Form farbloser, in Äther und Benzol löslicher Kristalle der analytischen Zusammensetzung $\mathrm{Al}\left(\mathrm{NR}_{2}\right)_{3}$ sammelt:

$$
\mathrm{AlH}_{3}+3 \mathrm{NHR}_{2} \rightarrow \mathrm{Al}\left(\mathrm{NR}_{2}\right)_{3}+3 \mathrm{H}_{2} .
$$

Die Substanz schmilzt bei $87-88^{\circ}$, wird von Wasser und Säuren zersetzt und ist in ätherischer Lösung nach der ebullioskopischen Mol.-Gew.-Bestimmung monomer (M gef.: 162; $\mathrm{M}$ ber.: 159). Die Einführung weiterer $\mathrm{NR}_{2}-$ Gruppen in das Alazen (4) bringt also dessen an sich schon schwache Polymerisationsneigung völlig zum Erliegen. Auch die homologe Borverbindung $\mathrm{B}\left(\mathrm{NR}_{2}\right)_{3}$ ist monomer ${ }^{3}$. -

Führt man die Umsetzung von Aluminiumwasserstoff mit überschüssigem Dimethylamin nicht bei Zimmertemperatur, sondern bei $-50^{\circ}$ durch und destilliert den Äther und das überschüssige Dimethylamin im Vakuum bei $-40^{\circ} \mathrm{ab}$, so hinterbleibt ohne Wasserstoffentwicklung ein Di-aminat

$$
\begin{aligned}
& \mathrm{H}>\mathrm{Al} \\
& \mathrm{NHR} \\
& \mathrm{NHR}_{2},
\end{aligned}
$$

das sich in seiner Zusammensetzung an die früher beschriebenen Di-aminate $\mathrm{AlH}_{3} \cdot 2 \mathrm{NR}_{3} 5, \mathrm{AlH}_{3} \cdot 2 \mathrm{NH}_{2} \mathrm{R} 6$ und $\mathrm{AlH}_{3} \cdot 2 \mathrm{NH}_{3}{ }^{7}$ anschließt. Es ist nicht sehr beständig und zerfällt in ätherischer Lösung schon oberhalb von $-20^{\circ}$ unter Abspaltung von Wasserstoff und Bildung der oben (Abschn. 2) beschriebenen Verbindung $\mathrm{AlH}\left(\mathrm{NR}_{2}\right)_{2}$ :

$$
\mathrm{AlH}_{3} \cdot 2 \mathrm{NHR}_{2} \rightarrow \mathrm{AlH}\left(\mathrm{NR}_{2}\right)_{2}+2 \mathrm{H}_{2} .
$$

Außer diesem Di-aminat (6) dürfte wie im Falle des Trimethylamins 5 , Methylamins ${ }^{6}$ und Ammoniaks 7 noch ein Mono-aminat $\mathrm{AlH}_{3} \cdot \mathrm{NHR}_{2}$ existieren, dessen Bildung der Entstehung von $\mathrm{AlH}_{2}\left(\mathrm{NR}_{2}\right)$ (Abschnitt 1) vorausgehen muß. -

Zusammenfassend kann gesagt werden, daß die auf analogem Wege wie die Borazene gewinnbaren Alazene an Polymerisationsfreudigkeit ihre leichteren Homologen übertreffen.

${ }^{6}$ E. Wiberg u. A. M ay, Z. Naturforschg. $10 \mathrm{~b}$, 232 [1955].

7 E. Wiberg u. A. May, Z. Naturforschg. 10 b, 229,230 [1955]. 